\title{
Existence and Uniqueness of a Fuzzy Solution for some Fuzzy Neutral Partial integro-Differential Equation with Nonlocal Conditions
}

\author{
Atimad HARIR $^{1}$, Said MELLIANI ${ }^{2}$ and Lalla Saadia CHADLI ${ }^{3}$ \\ 1,2,3 Laboratory of Applied Mathematics and Scientific Computing, Sultan Moulay Slimane University, \\ P.O. Box 523, Beni Mellal, 23000, Morocco \\ ${ }^{1}$ atimad.harir@gmail.com, ${ }^{2}$ melliani@fstbm.ac.ma, ${ }^{3}$ chadli@fstbm.ac.ma
}

\begin{abstract}
In this work, we establish several results about the existence of fuzzy solutions for some Fuzzy Neutral partial integro-Differential Equation with nonlocal condition. Our approach rest on the Banach fixed-point theorem.
\end{abstract}

Keywords: Neutral partial integro-Differential Equation, Fuzzy mild Solution, nonlocal conditions, fuzzy semigroups of linear operators

2010 MSC No: 34A07;35R13

\section{Introduction}

In this work, we study the existence of fuzzy mild solutions for fuzzy neutral partial integro-differential equations with nonlocal conditions of the following from:

$$
\left\{\begin{array}{l}
\frac{d}{d t}[x(t) \ominus F(t, x(t))] \oplus A x(t)=G(t, x(t)) \oplus \int_{0}^{t} H(t, s, x(a(s))) d s, \quad 0 \leq t \leq a, \\
x(0) \oplus g(x)=x_{0} \in E^{n},
\end{array}\right.
$$

where $A: E^{n} \rightarrow E^{n}$ is fuzzy operator, is the infinitesimal generator of an $C_{0}$-semigroup on $E^{n}$ and $E^{n}$ is the set of all upper semicontinuous, convex, normal fuzzy numbers with bounded a-level intervals, called spaces of fuzzy numbers, or more general with values in $E^{n}$, where $\left(E^{n}, \oplus, \odot, D\right)$ represents any from the fuzzy number type spaces introduced by section 2 , and $F, G:[0, T] \times E^{n} \rightarrow E^{n}, g: C\left([0, T] ; E^{n}\right) \rightarrow E^{n}, H:[0, T] \times[0, T] \times E^{n} \rightarrow E^{n}$ and $a \in C([0, T] ;[0, T])$.

Integro-differential equations play an important role in characterizing many social, physical, biological, and engineering problems. For example, Volterra was investigating the population growth, focusing his study on the hereditary influences, and several authors. see [1, 2] discussed the integro-differential modeled integral equations in the field of heat transfer and diffusion process in general neutron diffusion. Generally, several systems are mostly related to uncertainty and inexactness. The problem of inexactness is considered in general exact science, and that of uncertainty is considered as vague or fuzzy and accident. Ding and Kandel [3] analyzed a way to combine differential equations with fuzzy sets to form a fuzzy logic system called a fuzzy dynamical system, which can be regarded to form a fuzzy neutral functional differential equation.

Note that with respect to Banach spaces, the fuzzy number type spaces $\left(E^{n}, \oplus, \odot, D\right)$ represent more general structures, in the sense that although the metric has similar properties with a metric derived from a norm of Banach space, however $\left(E^{n}, \oplus, \odot, D\right)$ with respect to the addition $\oplus$ is not a group and with respect to the scalar multiplication is not linear space . 
The organization of this work is as follows:in Section 2, we call some fundamental results on fuzzy numbers. In Section 3 we study the existence of fuzzy mild solutions.

\section{Preliminaries}

In this section, we introduce notations, definitions, and preliminary facts which are used throughout this paper.

Let $T=[c, d] \subset \mathbb{R}$ be a compact interval and denote $E^{n}=\left\{u: \mathbb{R}^{n} \rightarrow[0,1] \mid u\right.$ satisfies (i)-(iv) below $\}$ where

(i) $\mathrm{u}$ is normal i.e, there exists an $x_{0} \in \mathbb{R}^{n}$ such that $u\left(x_{0}\right)=1$

(ii) $\mathrm{u}$ is fuzzy convex i.e for $x, y \in \mathbb{R}^{n}$ and $0<\lambda \leq 1$,

$$
u(\lambda x+(1-\lambda) y) \geq \min [u(x), u(y)]
$$

(iii) $\mathrm{u}$ is upper semicontinuous,

(iv) $[u]^{0}=\operatorname{cl}\left\{x \in \mathbb{R}^{n} \mid u(x)>0\right\}$ is compact.

For $0<\alpha \leq 1$ denote $[u]^{\alpha}=\left\{x \in \mathbb{R}^{n} \mid u(x) \geq \alpha\right\}$, then from (i) to (iv), it follows that the $\alpha$-level sets $[u]^{\alpha} \in P_{K}\left(\mathbb{R}^{n}\right)$ for all $0 \leq \alpha \leq 1$ is a closed bounded interval which we denote by $[u]^{\alpha}=\left[u_{l}^{\alpha}, u_{r}^{\alpha}\right]$.

Where $P_{K}\left(\mathbb{R}^{n}\right)$ denote the family of all nonempty compact convex subsets of $\mathbb{R}^{n}$. Define the addition and scalar multiplication in $P_{K}\left(\mathbb{R}^{n}\right)$ as usual.

If $g: \mathbb{R}^{n} \times \mathbb{R}^{n} \rightarrow \mathbb{R}^{n}$ is any function, then, according to Zadeh's extension principle, we can extend $g: E^{n} \times E^{n} \rightarrow E^{n}$ by the function defined by

$$
g(u, v)(z)=\sup _{z=g(x, y)} \min \{u(x), v(y)\}
$$

It is well known that

$$
[g(u, v)]^{\alpha}=g\left([u]^{\alpha},[v]^{\alpha}\right)
$$

for all $u, v \in E^{n}, 0 \leq \alpha \leq 1$ and a continuos function $g$. Especially for addition and scalar multiplication, we have $[u \oplus v]^{\alpha}=[u]^{\alpha}+[\bar{v}]^{\alpha},[\bar{k} \odot u]^{\alpha}=k[u]^{\alpha}$, where $u, v \in E^{n}, k \in \mathbb{R}, 0 \leq \alpha \leq 1$.

we say that there exists $a \ominus b$, if there exists $c \in E^{n}$ such that $a=b \oplus c$ and we denote $c=a \ominus b$ [4]

The distance between $\mathrm{A}$ and $\mathrm{B}$ is defined by the Hausdorff metric

$$
d_{H}(A, B)=\max \left\{\sup _{a \in A} \inf _{b \in B}\|a-b\|, \sup _{b \in B} \inf _{a \in A}\|a-b\|\right\}
$$

where $\|\cdot\|$ denotes the usual Euclidean norm in $\mathbb{R}^{n}$.

We define $D: E^{n} \times E^{n} \rightarrow \mathbb{R}^{+} \cup\{0\}$ by the equation

$$
D(u, v)=\sup _{\alpha \in[0,1]} d_{H}\left([u]^{\alpha},[v]^{\alpha}\right), \text { forall } u, v \in E^{n}
$$

where $d_{H}$ is the Hausdorff metric .

Then, from [5] Let $u, v, w$ and $e \in E^{n}$

(i) $D(u \oplus w, v \oplus w)=D(u, v)$

(ii) $D(k \odot u, k \odot v)=|k| D(u, v) \quad \forall k \in \mathbb{R}$

(iii) $D(u \oplus v, w \oplus e) \leq D(u, w)+D(v, e)$

(iv) If $u \odot v$ and $w \ominus e$ exist, then $D(u \ominus v, w \ominus e) \leq D(u, w)+D(v, e)$.

(v) $\left(E^{n}, D\right)$ is a complete metric space

Now, according to [6], with the aid of $\left(E^{n}, \oplus, \odot, D\right)$ we can define new spaces as follows. $C\left([0, a], E^{n}\right)$ the space of all continuous functions, endowed with the metric

$$
H(u, v)=\sup _{t \in T} D(u(t), v(t))
$$

and the natural operations induced by those in $E^{n},\left(C\left([0, a], E^{n}\right), H\right)$ is a complete metric space. [1] 
1. If we denote $\widetilde{0}=\chi_{\{0\}}$ then $\widetilde{0} \in E^{n}$ is neutral element with respect to $\oplus$ i.e $u \oplus \widetilde{0}=\widetilde{0} \oplus u=u$ for all $u \in E^{n}$

2. for any $\lambda, \mu \in \mathbb{R}$ with $\lambda, \mu \geq 0$ or $\lambda, \mu \leq 0$ and any $u, v \in E^{n}$ we have $(\lambda+\mu) \odot u=\lambda \odot u \oplus \mu \odot u$ for general $\lambda, \mu \in \mathbb{R}$ the above property does not hold

$\lambda \odot(u \oplus v)=\lambda \odot u \oplus \lambda \odot v, \lambda \odot(\mu \odot u)=(\lambda . \mu) \odot u$

3. If we denote $\|u\|_{E^{n}}=D(u, \widetilde{0}), \forall u \in E^{n}$ then $\|\cdot\|_{E^{n}}$ has the properties of a usual norm or $E^{n}$ i.e $\|.\|_{E^{n}}=0$ if $u=\widetilde{0},\|\lambda \odot u\|_{E^{n}}=|\lambda| \cdot\|u\|_{E^{n}}$ and $\|u \oplus v\|_{E^{n}} \leq\|u\|_{E^{n}}+\|v\|_{E^{n}}$ and $\|u\|_{E^{n}}-\|v\|_{E^{n}} \mid \leq D(u, v)$

from theorem 2 (2) we can deduce that for any $\lambda, \mu \in \mathbb{R}$ with $\lambda>\mu>0$ and any $u \in E^{n} \lambda \odot u \ominus \mu \odot u$ exists and $\lambda \odot u \ominus \mu \odot u=(\lambda-\mu) \odot u$ The following definitions and theorems are given in [7]

Definition 2.1. A mapping $F: T \times E^{n} \rightarrow E^{n}$ is strongly measurable if, for all $\alpha \in[0,1]$ the multi-valued mapping $F_{\alpha}: T \rightarrow P_{K}\left(\mathbb{R}^{n}\right)$ defined by

$$
F_{\alpha}(t)=[F(t)]^{\alpha}
$$

is Lebesgue measurable when $P_{K}\left(\mathbb{R}^{n}\right)$ is endowed with the topology generated by the Hausdorff metric $d_{H}$ and $T$ is a subinterval of real number $\mathbb{R}$.

Definition 2.2. A mapping is called levelwise continuous at $t_{0} \in T$ if the set-valued mapping $F_{\alpha}(t)=[F(t)]^{\alpha}$ is continuous at $t=t_{0}$ with respect to the Hausdorff metric $d_{H}$ for all $\alpha \in[0,1]$.

A mapping $F: T \rightarrow E^{n}$ is called integrably bounded if there exists an integrable function $h$ such that $\|x\| \leq h(t)$ for all $x \in F_{0}(t)$

Definition 2.3. Let $F: T \rightarrow E^{n}$. Then the integral of $F$ over $T$, denoted by $\int_{T} F(t) d t$ or $\int_{c}^{d} F(t) d t$ is defined

$$
\begin{aligned}
{\left[\int_{T} F(t) d t\right]^{\alpha} } & =\int_{T} F_{\alpha}(t) d t \\
& =\left\{\int_{T} f(t) d t \mid f: T \rightarrow \mathbb{R}^{n} \text { is a measurable selection for } F_{\alpha}\right\}
\end{aligned}
$$

for all $0<\alpha \leq 1$.

Also, a strongly measurable and integrably bounded mapping $F: T \times E^{n} \rightarrow E^{n}$ is said to be integrable over $T$ if $\int_{T} f(t) d t \in E^{n}$

If $F: T \rightarrow E^{n}$ is strangely mesurable and integrably bounded, then $\mathrm{F}$ is integrable. It is known that $\left[\int_{T} F(t) d t\right]^{0}=$ $\int_{T} F_{0}(t) d t$ [8] Let $F, G: T \rightarrow E^{n}$ be integrable and $\lambda \in \mathbb{R}$. Then

(i) $\int_{T}(F(t) \oplus G(t)) d t=\int_{T} F(t) d t \oplus \int_{T} G(t) d t$

(ii) $\int_{T} \lambda \odot F(t) d t=\lambda \odot \int_{T} F(t) d t$

(iii) $D(F, G)$ is integrable,

(iv) $D\left(\int_{T} F(t) d t, \int_{T} G(t) d t\right) \leq \int_{T} D(F, G)(t) d t$.

Definition 2.4. A mapping $F: T \rightarrow E^{n}$ is Hukuhara differentiable at $t_{0} \in T$ if there exists a $F^{\prime}\left(t_{0}\right) \in E^{n}$ such that the limits

$$
\lim _{h \rightarrow 0^{+}} \frac{F\left(t_{0}+h\right) \ominus F\left(t_{0}\right)}{h} \text { and } \lim _{h \rightarrow 0^{+}} \frac{F\left(t_{0}\right) \ominus F\left(t_{0}-h\right)}{h}
$$

exist and are equal to $F^{\prime}\left(t_{0}\right)\left(F^{\prime}\left(t_{0}\right)\right.$ is called the Hukuhara derivative of $F$ at $\left.t_{0} \in T\right)$. Here the limit is taken in the metric space $\left(E^{n}, D\right)$. At the end points of $T$, we consider only the one-site derivatives.

If $F: T \rightarrow E^{n}$ is differentiable at $t_{0} \in T$, then we say that $F^{\prime}\left(t_{0}\right)$ is the fuzzy derivative of $F(t)$ at point $t_{0}$. For the concepts of fuzzy measurability and fuzzy continuity we refer to [9]

Let $F: T \rightarrow E^{1}$ be differentiable with level sets $F_{\alpha}(t)=\left[f_{l}^{\alpha}, f_{r}^{\alpha}\right]$. Then $f_{l}^{\alpha}, f_{r}^{\alpha}:[0,1] \rightarrow \mathbb{R}^{1}$ are differentiable and $\left[F^{\prime}(t)\right]^{\alpha}=\left[f_{l}^{\alpha^{\prime}}, f_{r}^{\alpha^{\prime}}\right]$.see [10] 
Definition 2.5. A mapping $F: T \times E^{n} \rightarrow E^{n}$ is called levelwise continuous provided that for any fixed $\alpha \in[0,1]$ and arbitrary $\varepsilon>0$ there exists a $\delta(\varepsilon, \alpha)>0$ such that

$$
d_{H}\left([f(t, x)]^{\alpha},\left[f\left(t_{0}, x_{0}\right)\right]^{\alpha}\right)<\varepsilon,
$$

whenever $\left|t-t_{0}\right|<\delta(\varepsilon, \alpha)$ and $d_{H}\left([x]^{\alpha},\left[x_{0}\right]^{\alpha}\right)<\delta(\varepsilon, \alpha)$ for all $t \in T, x \in E^{n}$

Now, let us recall some elements of operator theory and semigroup of operators on $E_{n}$ in 6 ]

Definition 2.6. $A: E^{n} \rightarrow E^{n}$ is called linear operator if

$$
A(\lambda \odot x \oplus \mu \odot y)=\lambda \odot A(x) \oplus \mu \odot A(y)
$$

for all $\lambda, \mu \in \mathbb{R}$ and all $x, y \in E^{n}$ if

Definition 2.7. A family of functions $(T(t))_{t \geq 0}$ of continuous linear operators on $E^{n}$ is called fuzzy $C_{0}$-semigroup

1. For all $x \in E^{n}$ the mapping $T(t)(x): \mathbb{R}_{+} \rightarrow E^{n}$ is continuous with respect to $t \geq 0$

2. $T(t+s)=T(t)[T(s)]$ for all $t, s \in \mathbb{R}_{+}$

3. $T(0)=I$ where $I$ is the identity operator on $E^{n}$

Definition 2.8. if $A: E^{n} \rightarrow E^{n}$ is a linear operator, then it is called generator of the $C_{0}$-semigroup if for all $x \in E^{n}$, there exists $T(t)(x) \ominus x$ and

$$
\lim _{t \rightarrow 0^{+}} \frac{1}{t} \odot[T(t)(x) \ominus x]=A(x)
$$

6

(i) if $A: E^{n} \rightarrow E^{n}$ is linear and continuous on $\widetilde{0}$ then for all $x \in E^{n}$ we have

$$
\|A(x)\|_{E^{n}} \leq\|\| A\left\|\left.\right|_{E^{n}}\right\| x \|_{E^{n}}
$$

where \|\|$A\left\|\left.\right|_{E^{n}}=\sup \left\{\|A(x)\|_{E^{n}}, x \in E^{n},\|x\|_{E^{n}} \leq 1\right\} \in \mathbb{R}\right\| A(x) \|_{E^{n}}=D(A(x), \widetilde{0})$

if $\mathrm{A}$ is linear on $E^{n}$ and continuous on $\widetilde{0}$, then it does not follow the continuity of $\mathrm{A}$ on the whole space $E^{n}$

(ii) for any $\mathrm{A}$, linear and continuous operator on $E^{n}$, can be defined the linear and continuous operators $T(t)=$ $\exp (t \odot A), t \in \mathbb{R}$

$T(t)$ is differentiable with respect to $t \in \mathbb{R}_{+}$, with the derivative equal to $A[T(t)]$. More exactly, it is Hukuhara differentiable with respect to $t \in \mathbb{R}_{+}$i.e

$$
\begin{aligned}
& \lim _{h \rightarrow 0} D\left(\frac{1}{h} \odot(T(t+h)(x) \ominus T(t)(x)), A[T(t)(x)]\right)=0 \\
& \lim _{h \rightarrow 0} D\left(\frac{1}{h} \odot(T(t)(x) \ominus T(t-h)(x))=A[T(t)(x)]\right)=0
\end{aligned}
$$

By the linearity of $T(t)$ it easily follows that

$$
T(t)[x(t) \ominus y(t)]=T(t)[x(t)] \ominus T(t)[y(t)] \quad t \geq 0
$$

\section{Fuzzy Neutral partial integro-Differential Equation with nonlocal conditions}

Throughout the whole of this work, we assume that

$\left(H_{0}\right)$ The linear and continuous operator $A$ generates a $C_{0}$-semigroup $(T(t))_{t \geq 0}$ on $E^{n}$ such that

$$
\||T(t)|\|_{E^{n}} \leq M \text { for all } t \geq 0
$$


with $M>0$ Let $C\left([0, a], E^{n}\right)$ be the space of continuous functions. We assume that:

$\left(H_{1}\right) F, G:[0, a] \times E^{n} \rightarrow E^{n}$ are levelwise continuous and lipschitzians with respect to the second argument there exists constants $L_{1}>0$ and $L_{2}>0$ such that

$$
d_{H}\left([F(t, x)]^{\alpha},[F(t, y)]^{\alpha}\right) \leq L_{1} d_{H}\left([x]^{\alpha},[y]^{\alpha}\right)
$$

and

$$
d_{H}\left([G(t, x)]^{\alpha},[G(t, y)]^{\alpha}\right) \leq L_{2} d_{H}\left([x]^{\alpha},[y]^{\alpha}\right)
$$

for any pairs $(t, x),(t, y) \in[0, a] \times E^{n}$.

$\left(H_{2}\right) g: C\left([0, T], E^{n}\right) \rightarrow E^{n}$ is lipschitz continuous : there existe constants $L_{3}>0$ such that

$$
d_{H}\left(\left[g\left(u_{1}\right)\right]^{\alpha},\left[g\left(u_{2}\right)\right]^{\alpha}\right) \leq L_{3} d_{H}\left(\left[u_{1}\right]^{\alpha},\left[u_{2}\right]^{\alpha}\right)
$$

for $u_{1}, u_{2} \in C\left([0, T], E^{n}\right)$

$\left(H_{3}\right)$ There exists a constant 1 such that

$$
d_{H}\left([H(t, s, x(s))]^{\alpha},[H(t, s, y(s))]^{\alpha}\right) \leq l d_{H}\left([x]^{\alpha},[y]^{\alpha}\right)
$$

for any pairs $(t, s, x),(t, s, y) \in[0, T] \times[0, T] \times E^{n}$.

$\left(H_{4}\right) a: C([0, T],[0, T])$

Definition 3.1. A continuous function $x():.[0, T] \rightarrow E^{n}$ is said to be a mild solution of equation (1) if

(a) $A T(t-s) F(s, x(s))$ is integrable

(b)

$$
\begin{aligned}
x(t) & =T(t)\left[x_{0} \ominus g(x) \ominus F(0, x(0))\right] \oplus F(t, x(t)) \oplus \int_{0}^{t} A T(t-s) F(s, x(s)) d s \\
& \oplus \int_{0}^{t} T(t-s) G(s, x(s)) d s \oplus \int_{0}^{t} T(t-s)\left(\int_{0}^{s} H(s, \tau, x(a(\tau))) d \tau\right) d s
\end{aligned}
$$

for all $t \in[0, T]$

everywhere in this section when we refer to the equation (1) we mean that there + is replaced by the fuzzy addition $\oplus$ and - is replaced by the fuzzy subtraction $\ominus$. Assume that assumptions $\left(H_{0}\right)-\left(H_{4}\right)$ hold. Then there existe a unique mild solution $x=x(t)$ of Eq (1) provided that $L_{0}=M L_{3}+\eta L_{1}+T M L_{2}+M T^{2} l<1$ where $\eta=M+1+M \mid\|A\| \|_{E^{n}} T$

Proof. Consider the operator $\psi$ defined on $C\left([0, T] ; E^{n}\right)$ by

$$
\begin{aligned}
(\psi x)(t) & =T(t)\left[x_{0} \ominus g(x) \ominus F(0, x(0))\right] \oplus F(t, x(t)) \oplus \int_{0}^{t} A T(t-s) F(s, x(s)) d s \\
& \oplus \int_{0}^{t} T(t-s) G(s, x(s)) d s \oplus \int_{0}^{t} T(t-s)\left(\int_{0}^{s} H(s, \tau, x(a(\tau))) d \tau\right) d s
\end{aligned}
$$

for all $0 \leq t \leq T$ we shall that is $\psi$ a contraction operator. Indeed, consider $x, y \in C\left([0, T] ; E^{n}\right)$ and $\alpha \in(0,1]$, then

$$
\begin{aligned}
D((\psi x)(t),(\psi y)(t))= & D\left(\left[T(t)\left[x_{0} \ominus g(x) \ominus F(0, x(0))\right] \oplus F(t, x(t)) \ominus \int_{0}^{t} A T(t-s) F(s, x(s)) d s\right.\right. \\
& \left.\oplus \int_{0}^{t} T(t-s) G(s, x(s)) d s \oplus \int_{0}^{t} T(t-s)\left(\int_{0}^{s} H(s, \tau, x(a(\tau))) d \tau\right) d s\right] \\
& ,\left[T(t)\left[x_{0} \ominus g(y) \ominus F(0, y(0))\right] \oplus F(t, y(t)) \ominus \int_{0}^{t} A T(t-s) F(s, y(s)) d s\right. \\
& \left.\left.\oplus \int_{0}^{t} T(t-s) G(s, y(s)) d s \oplus \int_{0}^{t} T(t-s)\left(\int_{0}^{s} H(s, \tau, y(a(\tau))) d \tau\right) d s\right]\right) \\
& \leq D([T(t) g(x)],[T(t) g(y)])+D([T(t) F(0, x(0))],[T(t) F(0, y(0))])
\end{aligned}
$$




$$
\begin{aligned}
& +D(F(t, x(t)), F(t, y(t)))+D\left(\left[\int_{0}^{t} A T(t-s) F(s, x(s)) d s\right],\left[\int_{0}^{t} A T(t-s) F(s, y(s)) d s\right]\right) \\
& +D\left(\left[\int_{0}^{t} T(t-s) G(s, x(s)) d s\right],\left[\int_{0}^{t} T(t-s) G(s, y(s)) d s\right]\right) \\
& +D\left(\left[\int_{0}^{t} T(t-s)\left(\int_{0}^{s} H(s, \tau, x(a(\tau))) d \tau\right) d s\right],\left[\int_{0}^{t} T(t-s)\left(\int_{0}^{s} H(s, \tau, y(a(\tau))) d \tau\right) d s\right]\right) \\
& \leq D([T(t) g(x)],[T(t) g(y)])+D([T(t) F(0, x(0))],[T(t) F(0, y(0))]) \\
& +D(F(t, x(t)), F(t, y(t)))+\int_{0}^{t} D([A T(t-s) F(s, x(s))],[A T(t-s) F(s, y(s))]) d s \\
& +\int_{0}^{t} D([T(t-s) G(s, x(s))],[T(t-s) G(s, y(s))]) d s \\
& +\int_{0}^{t} D\left(\left[T(t-s)\left(\int_{0}^{s} H(s, \tau, x(a(\tau))) d \tau\right)\right],\left[\int_{0}^{t} T(t-s)\left(\int_{0}^{s} H(s, \tau, y(a(\tau))) d \tau\right)\right]\right) d s
\end{aligned}
$$

$$
\begin{aligned}
& =D([T(t) g(x)], \widetilde{0})+D(\widetilde{0},[T(t) g(y)])+D([T(t) F(0, x(0))], \widetilde{0}) \\
& +D(\widetilde{0},[T(t) F(0, y(0))])+D(F(t, x(t)), F(t, y(t))) \\
& +\int_{0}^{t}[D([A T(t-s) F(s, x(s))], \widetilde{0})+D([A T(t-s) F(s, y(s))], \widetilde{0})] d s \\
& +\int_{0}^{t}[D([T(t-s) G(s, x(s))], \widetilde{0})+D([T(t-s) G(s, y(s))], \widetilde{0})] d s \\
& +\int_{0}^{t}\left[D\left(\left[T(t-s)\left(\int_{0}^{s} H(s, \tau, x(a(\tau))) d \tau\right)\right], \widetilde{0}\right)+D\left(\left[T(t-s)\left(\int_{0}^{s} H(s, \tau, y(a(\tau))) d \tau\right)\right], \widetilde{0}\right)\right] d s \\
& \leq\left.\quad\|T(t)\|\right|_{E^{n}} D(g(x), g(y))+\mid\|T(t)\| \|_{E^{n}} D(F(0, x(0)), F(0, y(0))) \\
& +\quad D(F(t, x(t)), F(t, y(t))) \\
& +\int_{0}^{t}\left[\|\| T(t-s)\|\|_{E^{n}} D(A F(s, x(s)), \widetilde{0})+D(\widetilde{0}, A F(s, y(s))) d s\right. \\
& +\int_{0}^{t}\left[\|T(t-s)\| \|_{E^{n}} D(G(s, x(s)), G(s, y(s)))\right] d s \\
& +\int_{0}^{t}\left[\|T(t-s)\| \|_{E^{n}} D\left(\left(\int_{0}^{s} H(s, \tau, x(a(\tau))) d \tau\right),\left(\int_{0}^{s} H(s, \tau, y(a(\tau))) d \tau\right)\right)\right] d s \\
& \leq M D(g(x), g(y))+M D(F(0, x(0)), F(0, y(0)))+D(F(t, x(t)), F(t, y(t))) \\
& +M \mid\|A\| \|_{E^{n}} \int_{0}^{t}\left[D(F(s, x(s)), F(s, y(s))) d s+M \int_{0}^{t}[D(G(s, x(s)), G(s, y(s)))] d s\right. \\
& +M \int_{0}^{t}\left[D\left(\left(\int_{0}^{s} H(s, \tau, x(a(\tau))) d \tau\right),\left(\int_{0}^{s} H(s, \tau, y(a(\tau))) d \tau\right)\right)\right] d s
\end{aligned}
$$




$$
\begin{aligned}
& =M \sup _{\alpha[0,1]}\left\{d_{H}\left([g(x)]^{\alpha},[g(y)]^{\alpha}\right)\right\} \\
& +\quad M \sup _{\alpha[0,1]}\left\{d_{H}\left([F(0, x(0))]^{\alpha},[F(0, y(0))]^{\alpha}\right)\right\}+\sup _{\alpha[0,1]}\left\{d_{H}\left([F(t, x(t))]^{\alpha},[F(t, y(t))]^{\alpha}\right)\right\} \\
& +\left.\quad M\|A\|\right|_{E^{n}} \int_{0}^{t} \sup _{\alpha[0,1]}\left\{d_{H}\left([F(t, x(t))]^{\alpha},[F(t, y(t))]^{\alpha}\right)\right\} d s \\
& +M \int_{0}^{t} \sup _{\alpha[0,1]}\left\{d_{H}\left([G(s, x(s))]^{\alpha},[G(s, y(s))]^{\alpha}\right)\right\} d s \\
& +\quad M \int_{0}^{t} \sup _{\alpha[0,1]}\left\{\left[d_{H}\left(\left[\int_{0}^{s} H(s, \tau, x(a(\tau))) d \tau\right]^{\alpha},\left[\int_{0}^{s} H(s, \tau, y(a(\tau))) d \tau\right]^{\alpha}\right]\right)\right\} d s \\
& \leq \quad M L_{3} \sup _{\alpha[0,1]}\left\{d_{H}\left([x]^{\alpha},[y]^{\alpha}\right)\right\} \\
& +\quad M L_{1} \sup _{\alpha[0,1]}\left\{d_{H}\left([x]^{\alpha},[y]^{\alpha}\right)\right\}+L_{1} \sup _{\alpha[0,1]}\left\{d_{H}\left([x]^{\alpha},[y]^{\alpha}\right)\right\} \\
& +\quad M L_{1}\|\| A \|\left.\right|_{E^{n}} \int_{0}^{t} \sup _{\alpha[0,1]}\left\{d_{H}\left([x]^{\alpha},[y]^{\alpha}\right)\right\} d s+M L_{2} \int_{0}^{t} \sup _{\alpha[0,1]}\left\{d_{H}\left([x]^{\alpha},[y]^{\alpha}\right)\right\} d s \\
& +M \int_{0}^{t} T l \sup _{\alpha[0,1]}\left\{d_{H}\left([x]^{\alpha},[y]^{\alpha}\right)\right\} d s \\
& \leq M L_{3} D(x(t), y(t))+M L_{1} D(x(t), y(t))+L_{1} D(x(t), y(t)) \\
& +M L_{1}|\|A\||_{E^{n}} \int_{0}^{t} D(x(s), y(s)) d s+M L_{2} \int_{0}^{t} D(x(s), y(s)) d s+M T l \int_{0}^{t} D(x(s), y(s)) d s
\end{aligned}
$$

Then we obtain

$$
\begin{aligned}
D((\psi x)(t),(\psi y)(t)) \leq & M L_{3} D(x(t), y(t))+M L_{1} D(x(t), y(t))+L_{1} D(x(t), y(t)) \\
& +M L_{1} \mid\|A\|_{E^{n}} \int_{0}^{t} D(x(s), y(s)) d s+M L_{2} \int_{0}^{t} D(x(s), y(s)) d s \\
& +M T l \int_{0}^{t} D(x(s), y(s)) d s \\
H(\psi x, \psi y) \leq & \sup _{0 \leq t \leq T}\left\{M L_{3} D(x(t), y(t))+L_{1}(M D(x(t), y(t))+D(x(t), y(t))\right. \\
+ & \left.M \mid\|A\| \|_{E^{n}} \int_{0}^{t} D(x(s), y(s)) d s\right)+M L_{2} \int_{0}^{t} D(x(s), y(s)) \\
& \left.+M T l \int_{0}^{t} D(x(s), y(s)) d s\right\} \\
\leq & \left(M L_{3}+\left(M+1+M|\|A\||_{E^{n}} T\right) L_{1}+T M L_{2}+M T^{2} l\right) H(x, y)
\end{aligned}
$$

Since $M L_{3}+\eta L_{1}+T M L_{2}+M T^{2} l<1$ where $\eta=M+1+M|\|A\||_{E^{n}} T, \psi$ is a strict contraction mapping. By Banach fixed point theorem we conclude that $\psi$ has a unique fixed point $x=\psi x \in C\left([0, a], E^{n}\right)$

\section{References}

[1] K. Ezzinbi and X. Fu, Existence and regularity of solutions for some neutral partial differential equations with nonlocal conditions, Nonlinear Analysis, 57 (2004) 1029-1041 https://doi.org/10.1016/j.na.2004.03.027 
[2] Y. Lin and H. Liu, Semilinear integrodifferential equations with nonlocal Cauchy problem, Nonlinear Anal. Theory Methods Appl. 26 (1996) 1023-1033.

[3] Z. Ding and A. Kandel, On the controllability of fuzzy dynamical systems (I), J. Fwzy Maths., 8 (2000), $203-214$.

[4] D. Dubois and Prade, fuzzy numbers; on overview in : Analysis of fuzzy Information, vol.1 Math .logic, CRC press, boca Raton, 1987,pp.3-29 https://doi.org/10.1016/b978-1-4832-1450-4.50015-8

[5] V. Lakshmikantham, T. GnanaBhaskar and J. VasundharaDevi, Theory of Set Differential Equations in Metric Spaces, Cambridge Scientific Publishers, 2006.

[6] C.S. Gal and S.G. Gal, Semigroups of operators os spaces of fuzzy-number-valued functions with applications to fuzzy differential equations, J. FuzzyMath. 13(2005), 647-682.

[7] O. Kaleva, Fuzzy differential equations Fuzzy Sets and Systems, 24 (1987) 301-317. https://doi.org/10.1016/01650114(87)90029-7

[8] C. Wu, S. Song and E. StanleyLee, Approximatesolutions, existence, and uniqueness of the Cauchy problem of fuzzy differential equations, J. Math.Anal.Appl.202(1996), 629-644. https://doi.org/10.1016/s0165-0114(97)003990

[9] Y. K. Kim, Measurability for fuzzy valued functions. Fuzzy Sets and Systems, 129(1)(2002), $105-109$. https://doi.org/10.1016/s0165-0114(01)00121-x

[10] L. S. Chadli, A. Harir and S. Melliani, Fuzzy Euler Differential Equation, SOP Trans. Appli. Math., 2(1) (2015). https://doi.org/10.15764/am.2015.01001 